\title{
Hyperbaric Oxygen Therapy: Solution for Difficult to Heal Acute Wounds? Systematic Review
}

\author{
Anne M. Eskes • Dirk T. Ubbink • \\ Maarten J. Lubbers $\cdot$ Cees Lucas • \\ Hester Vermeulen
}

Published online: 24 December 2010

(C) The Author(s) 2010. This article is published with open access at Springerlink.com

\begin{abstract}
Background Hyperbaric oxygen therapy (HBOT) is used to treat various wound types. However, the possible beneficial and harmful effects of HBOT for acute wounds are unclear. Methods We undertook a systematic review to evaluate the effectiveness of HBOT compared to other interventions on wound healing and adverse effects in patients with acute wounds. To detect all available randomized controlled trials (RCTs) we searched five relevant databases up to
\end{abstract}

This article is based on a Cochrane review first published in The Cochrane Library 2010, Issue 10 (see http://www.thecochranelibrary. $\mathrm{com} /$ for information). Cochrane reviews are regularly updated as new evidence emerges and in response to feedback; and The Cochrane Library should be consulted for the most recent version of the review. The results of a Cochrane review can be interpreted differently, depending on people's perspectives and circumstances. Please consider the conclusions presented carefully. They are the opinions of review authors and are not necessarily shared by The Cochrane Collaboration. This systematic review has been prepared under the aegis of The Cochrane Collaboration, an international organization that aims to help people make well-informed decisions about health care by preparing, maintaining, and promoting the accessibility of systematic reviews of the effects of health care interventions. The Collaboration's publication policy permits journals to publish reviews, with priority if required, but permits The Cochrane Collaboration also to publish and disseminate such reviews. Cochrane reviews cannot be subject to the exclusive copyright requested by some journals.

A. M. Eskes · D. T. Ubbink $(\bowtie) \cdot H$. Vermeulen

Department of Quality Assurance \& Process Innovation,

Room A3-503, Academic Medical Center, University

of Amsterdam, Meibergdreef 9, P.O. Box 22700,

Amsterdam 1100 DE, The Netherlands

e-mail: d.ubbink@amc.nl

A. M. Eskes - H. Vermeulen

Amsterdam School of Health Professions,

Amsterdam, The Netherlands
March 2010. Trial selection, quality assessment, data extraction, and data synthesis were conducted by two of the authors independently.

Results We included five trials, totaling 360 patients. These trials, with some methodologic flaws, included different kinds of wound and focused on different outcome parameters, which prohibited meta-analysis. A French trial ( $n=36$ patients) reported that significantly more crush wounds healed with HBOT than with sham HBOT [relative risk (RR) 1.70, 95\% confidence interval (CI) 1.11-2.61]. Moreover, there were significantly fewer additional surgical procedures required with HBOT (RR 1.60, 95\% CI $1.03-2.50$ ), and there was significantly less tissue necrosis (RR 1.70, 95\% CI 1.11-2.61). In one of two American trials $(n=141)$ burn wounds healed significantly quicker with HBOT $(P<0.005)$ than with routine burn care. A British trial $(n=48)$ compared HBOT with usual care. HBOT resulted in a significantly higher percentage of healthy graft area in split skin grafts (RR 3.50, 95\% CI 1.35-9.11). In a Chinese trial $(n=145)$ HBOT did not significantly improve flap survival in patients with limb skin defects.

Conclusions HBOT, if readily available, appears effective for the management of acute, difficult to heal wounds.

\section{T. Ubbink · M. J. Lubbers}

Department of Surgery, Academic Medical Center,

University of Amsterdam, Amsterdam,

The Netherlands

\section{Lucas}

Department of Clinical Epidemiology, Biostatics, and Bioinformatics, Academic Medical Center,

University of Amsterdam, Amsterdam,

The Netherlands 


\section{Introduction}

Acute wounds are characterized by breakdown of the integrity of the soft tissue envelope surrounding any portion of the body [1] and are mostly subsequent to a surgical intervention or trauma. Such wounds occur more frequently than chronic wounds [2]. Most acute wounds heal without difficulty. However, some are subject to factors that impede healing, such as poor local blood and oxygen supply, infection, or damage to the vasculature. If one of these complicating factors occurs, these wounds can be considered potential problem wounds with an extended healing time. In these cases, more specific care is needed.

Hyperbaric oxygen therapy (HBOT)-delivering $100 \%$ oxygen at pressures above one atmosphere-is thought to assist wound healing, as it delivers a significantly increased amount of oxygen to the skin and surrounding tissues $[3,4]$. For this reason, HBOT has been used as a treatment for various chronic and acute wounds [5-7]. HBOT was shown to be effective for patients with diabetic foot ulcers, and it significantly reduced the risk of major amputation and improved the chance of healing at 1 year $[6,8]$. Two other Cochrane systematic reviews showed that the potential value of HBOT for open fractures and burns was unclear [7, 9]. Another literature review by Goldman showed some evidence that HBOT promotes healing of arterial ulcers, calciphylactic ulcers, refractory vasculitic ulcers, and osteomyelitis, as well as the successful "take" of compromised flaps and grafts [10]. However, that review included randomized clinical trials (RCTs), nonrandomized trials (CCTs), cohort studies, retrospective analyses, and case series. Inclusion of the latter study designs and the fact it was a single-author review may produce results that are at a considerable risk of bias.

Evidence regarding the effectiveness of HBOT for acute wounds has never been appreciated systematically. We therefore carried out a Cochrane systematic review to answer the following question: Is HBOT more effective than other types of treatment (e.g., usual care, sham HBOT, different intensities of HBOT, or different numbers of treatment applications) for acute wounds in terms of wound healing, adverse effects, pain, and cost?

\section{Methods}

Protocol and registration

Methods of the analysis and inclusion criteria were specified in advance and documented in a protocol described elsewhere [11]. Here, our methods and results are summarized according to the Preferred Reporting Items for Systematic Reviews and Meta-Analyses (PRISMA) statement [12, 13].
Eligibility criteria

In this review, we included all RCTs that evaluated the effects of HBOT as treatment for acute wounds in adult patients. These RCTs compared HBOT with any other intervention or sham HBOT, or they make comparisons between different intensities of HBOT or the use different numbers of treatment applications. We included such RCTs if they assessed at least one of the following primary outcome parameters: wound healing and adverse effects. As secondary outcome parameters, we recorded mortality, pain scores, quality of life, patient satisfaction, activities of daily living, increase in transcutaneous oxygen pressure $(\mathrm{tcPO})$, amputation rate, length of hospital stay, and cost.

Information sources

The RCTs were identified by searching the following databases: Cochrane Wounds Group Specialised Register, Ovid Medline, Ovid Embase, and EBSCO Cinahl up to March 2010, and the Cochrane Central Register of Controlled Trials (CENTRAL) up to issue 1, 2010. The search strategy is detailed elsewhere [11]. Furthermore, we used our wound expert network to check for ongoing or unpublished trials. We also screened the reference lists of all included articles to identify additional relevant trials. We used no restriction on language, publication data, or publication status.

\section{Study selection}

Two review authors independently selected potentially relevant trials based on the titles and abstracts of the articles retrieved by the search. These authors were not masked for any information about the article, such as the publishing journal, the authors, the institution, or the magnitude or direction of the results.

Full-text versions of the articles were obtained if they matched the inclusion criteria or if further scrutiny was needed about eligibility. The final selection of trials to be included was made independently by the same review authors. A third review author was involved in case of any discrepancies.

\section{Data collection process}

Two authors independently extracted and summarized characteristics and data from the included trials. Disagreements were resolved by discussion between the two review authors; if no agreement could be reached, a third author would decide. We contacted one author to obtain missing information but received no response. Data from trials published in duplicate were included only once. 
Risk of bias in individual studies

The methodologic quality of each trial was determined by two of the authors independently. We used the Cochrane Collaboration appraisal tool to assess risk of bias [14]. This involves consideration of six quality items: sequence generation, allocation concealment, blinding, incomplete outcome data, selective reporting, and "other" potential sources of bias [14]. Again, a third review author arbitrated any discrepancies.

\section{Data items}

From each included study, information was extracted about (1) characteristics of trial participants and inclusion and exclusion criteria; (2) type of intervention (including type, dose, duration, and frequency of the treatment) versus control intervention; (3) type of outcome.

Summary measures and methods of analysis

Quantitative data were entered and analyzed in RevMan 5.0.24 (Cochrane Collaboration, Oxford, UK) by one author and checked by another. We calculated summary estimates of the treatment effects with $95 \%$ confidence intervals $(95 \%$ CI) for every comparison. For continuous outcome parameters, we calculated the mean differences (MD) when appropriate. For dichotomous outcome parameters we calculated the risk ratio (RR). To present the number needed to treat (NNT), the risk difference (RD) was calculated.

\section{Results}

\section{Study selection}

The search provided a total of 137 publications. The study inclusion process is shown in Fig. 1. Five RCTs fulfilled our inclusion criteria. No new RCTs were found by reference checking. Also, no unpublished relevant RCTs were obtained.

\section{Characteristics of included studies}

\section{Participants and intervention}

Four of the five included trials were in English. One trial was translated from Chinese into Dutch by a national translation institute. Trials ranged in size from 16 to 135 patients, totaling 360 patients. HBOT was applied for various indications: crush injuries [15], burns [16, 17], split-skin grafts (SSG) [18], and flap grafting for limb skin defects [19]. The type of HBOT chamber and the duration and dose of the treatment were different among the trials. In addition, HBOT was compared to other treatments (Table 1).

\section{Primary and secondary outcomes}

In four trials the primary outcome assessed was wound healing, although measured differently. In one trial length of hospital stay was described as the primary outcome [16]. Adverse effects (including new surgical procedures and amputation rates) and length of hospital stay were described in only two trials $[15,16]$. Mortality was described twice $[16,17]$. Other outcomes were not reported (Table 1).

Risk of bias within studies

The methodologic quality of the trials was moderate. Hence, their internal validity was reasonable (Table 2). Only one of the trials reported their method of randomization [17]. As a consequence, we could not appreciate allocation concealment in the other trials. Bouachour et al. [15] and Hart et al. [17] blinded care provider, patients, and outcome assessor to treatment allocation. Patients and care providers were not blinded in the trial of Brannen et al., and blinding of the outcome assessor was unclear [16]. Perrins blinded the surgeon to treatment allocation [18] and Xie and $\mathrm{Li}$ reported that no one was blinded [19]. All randomized participants were analyzed in the group to which they had been allocated in the trials of Bouachour et al. [15] and Xie and Li [19]. However, in Perrins' trial two patients were excluded from the analysis [18]. Nevertheless, we were able to perform an intention-to-treat analysis post hoc. Furthermore, in the trials of Brannen et al. [16] and Hart et al. [17], it was unclear if they used an intention to treat-analysis for all the endpoints.

Hart et al. [17], Perrins [18], and Xie and Li [19] did not report the characteristics of the participants at baseline. Hence, we were unable to judge baseline comparability. Bouachour et al. [15] and Hart et al. [17] were supported by a research grant received from their hospital but had no financial conflict of interest with respect to the trial outcome.

\section{Quantitative data}

The trials varied markedly in terms of wound types, comparator treatments, and outcome parameters. Therefore, the clinical heterogeneity was substantial, so we focused on describing individual trial results, applicability, and limitations rather than using a meta-analysis.

\section{Crush injuries}

Bouachour et al. [15] studied HBOT in patients with crush injuries [15]. In this French trial, complete wound healing 
Fig. 1 Flow of information through the various phases of a systematic review

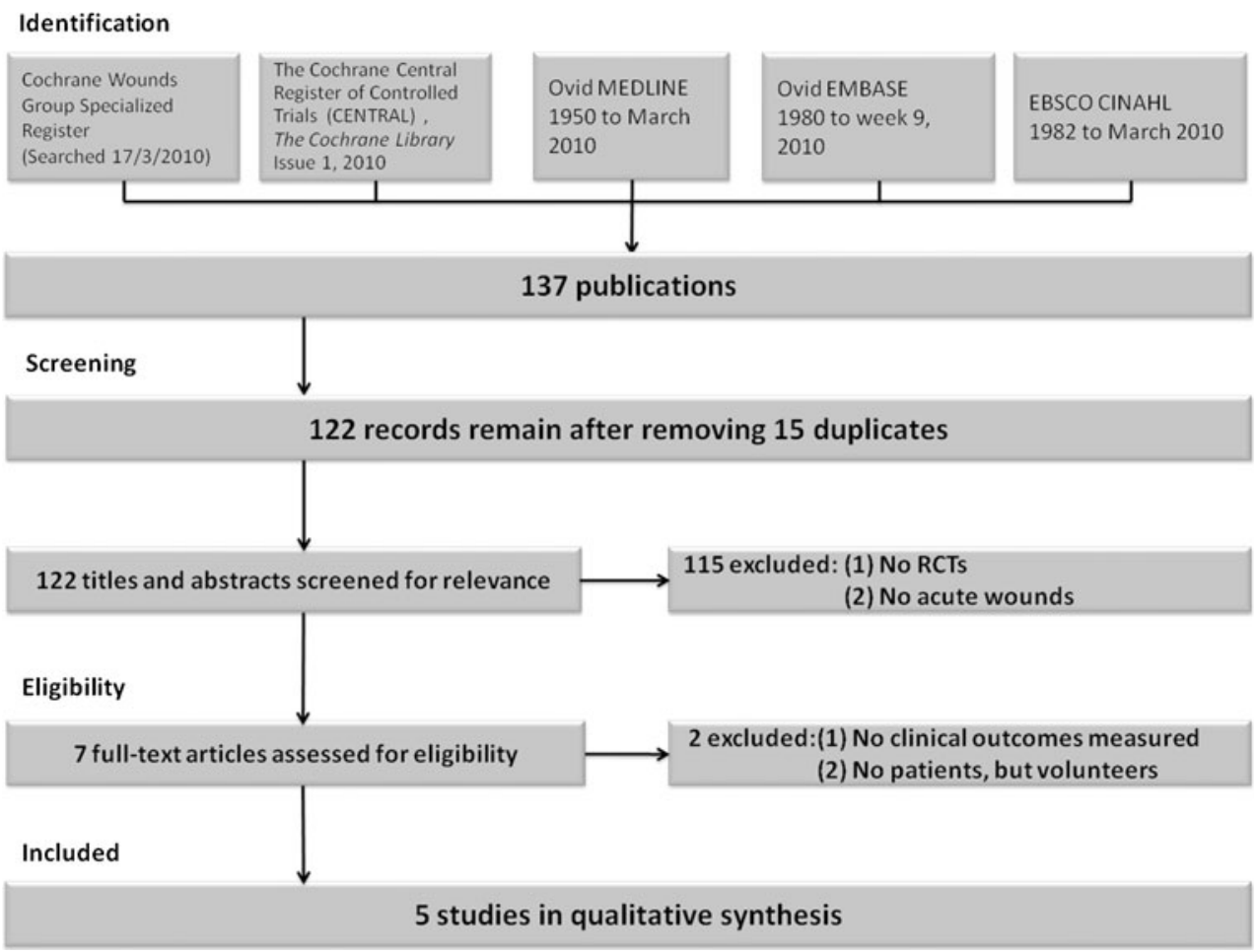

(without tissue necrosis requiring surgical excision) was achieved in 17 patients $(94 \%)$ treated with HBOT versus 10 patients $(56 \%)$ in the sham-HBOT group, showing a statistically significant difference in favor of HBOT therapy (RR 1.70; 95\% CI 1.11-2.61; NNT 3). However, they found no statistical difference between the two groups in time to complete healing (mean $\pm \mathrm{SD}$ ) $50.2 \pm 21.1$ days in the HBOT group versus $55.8 \pm 19.9$ days in the shamHBOT group (MD 5.60; 95\% CI 19.00-7.80).

Bouachour et al. [15] also compared HBOT with shamHBOT in terms of adverse effects. Two additional surgical procedures (in one patient) were needed in the HBOT group versus eight (in six patients) in the sham-HBOT group (RR 1.60, 95\% CI 1.03-2.50; NNT 3), as the first operation did not have the desired effect. In addition, one patient in the HBOT group versus eight patients in the sham-HBOT group developed necrotic tissue (RR 1.70, 95\% CI 1.11-2.61; NNT 3). No amputations occurred in the HBOT group versus two amputations in the shamHBOT group (RR 1.12, 95\% CI 0.93-1.36) and no significant differences were observed between the two groups for length of hospital stay (MD 0.50; 95\% CI 9.96-8.96). Other (secondary) endpoints were not reported.

\section{Burns}

Brannen et al. [16] and Hart et al. [17] studied HBOT in burn patients in the United States. Brannen et al. did not study wound healing but found no significant differences in mortality rates between HBOT and routine burn management (RR 0.98, 95\% CI 0.37-2.64). Also, no significant differences in length of hospital stay or number of surgeries were found. However, we did not have access to the original data and therefore could not reanalyze it. Hart et al. reported a significantly lower mean healing time in the HBOT-treated group (mean 19.7 days) than in the sham-HBOT group (mean 43.8 days) $(P<0.005)$. No SDs were given to check this result. No deaths were observed. Three patients in the HBOT group experienced sinus barotrauma, and one patient in the control group had transient viremia during the treatment.

\section{Split-skin grafts}

Perrins studied HBOT applied to patients who had undergone a split-skin graft (SSG) [18]. In this British trial, complete survival was defined as $95 \%$ take of the graft. Patients treated with HBOT had a significantly higher percentage complete survival ( $>95 \%$ healthy graft area: RR 3.50; 95\% CI 1.35-9.11; NNT 2). Consistently, he found better results in the HBOT group, but two grafts (8\%) in the HBOT group failed completely versus no failures in the control group (RR 5.00; 95\% CI 0.25-98.96). However, this was not significantly different. Other secondary outcomes were not reported for these SSGs.

\section{Flap grafting}

Xie and Li studied HBOT in patients with skin defects in the limbs, for which they underwent flap grafting [19]. In 


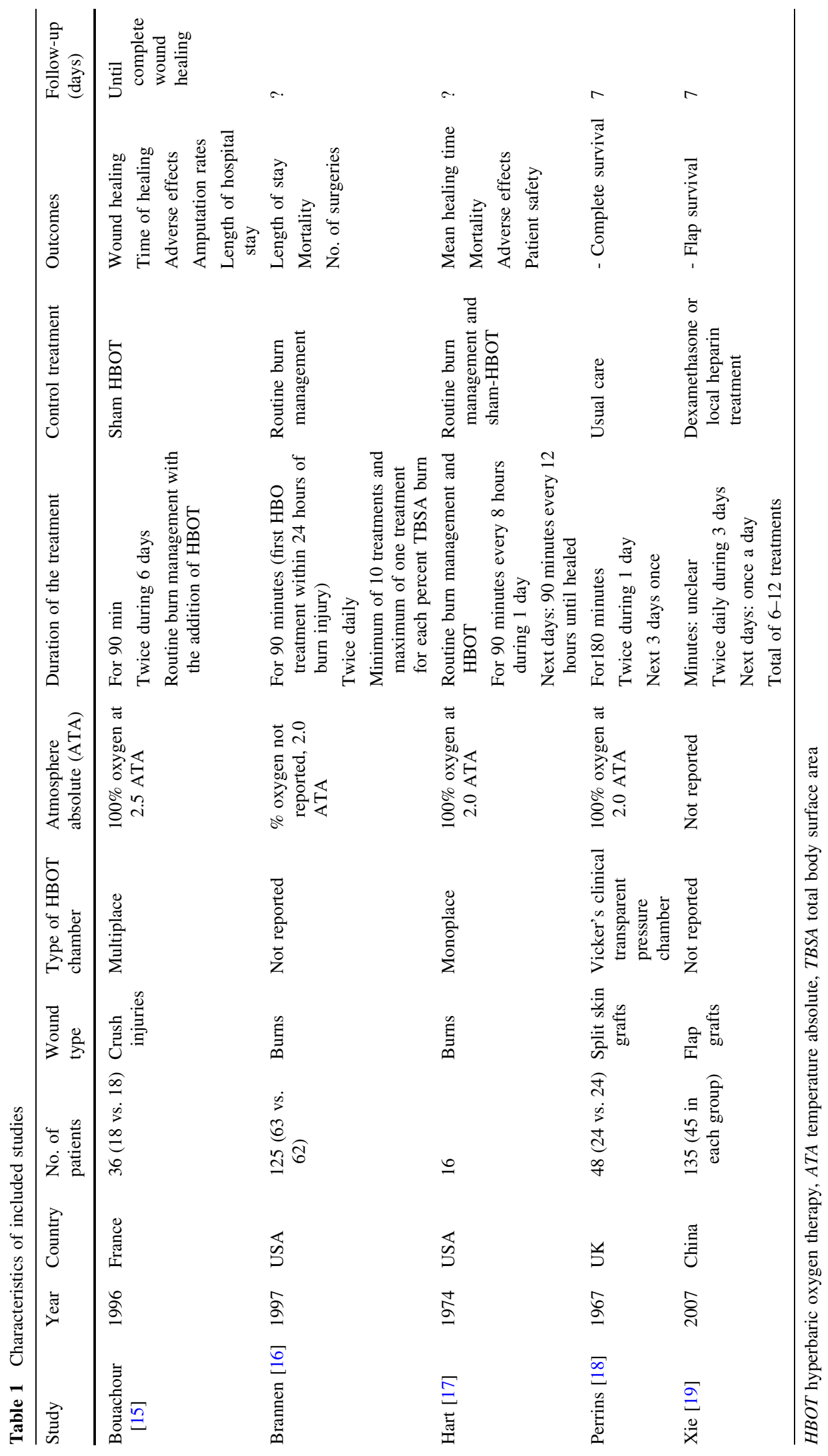




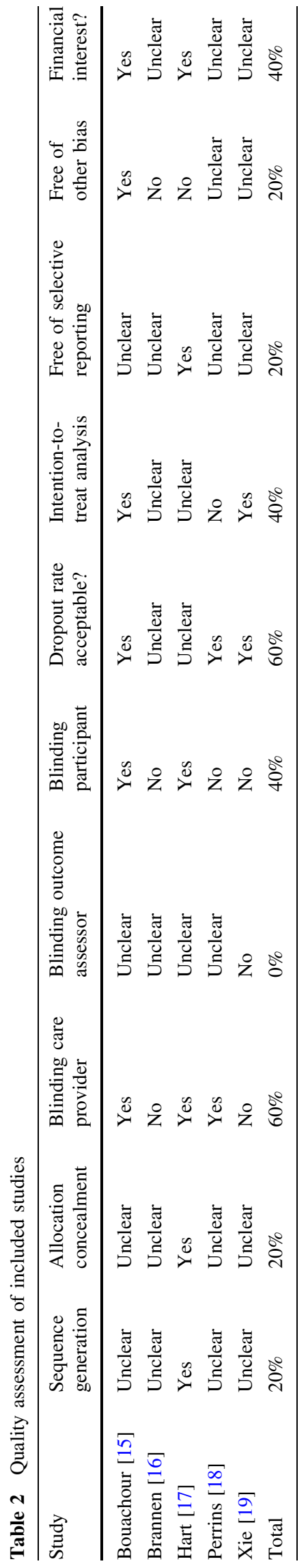

this Chinese trial, no significant differences in complete flap survival were found between HBOT and dexamethasone (RR 0.50, 95\% CI 0.19-1.35) and between HBOT and heparin (RR 0.42, 95\% CI 0.16-1.09).

\section{Discussion}

This review shows that some evidence is available based on robust study designs regarding the effectiveness of HBOT for the management of acute wounds, although the trials found were few and showed several methodologic flaws. Only five RCTs were found, the results of which could not be pooled. However, based on individual trial data, HBOT appears beneficial in patients with crush injuries, which show improved wound healing and less adverse outcomes such as necrosis or the need for additional surgical procedures. Also, burns appear to heal quicker with HBOT. Furthermore, HBOT appears to improve the take of split-skin grafts. On the other hand, complete wound healing is the most patient-relevant outcome. Hence, incomplete graft take, leading to a mere reduction in wound size, is not very meaningful.

Our findings are supported by those of previous reviews by Goldman [10] and Wang et al. [5]. Their reviews included studies with designs other than RCTs. Additionally, burns were not included. They concluded that HBOT may be beneficial as an adjunctive therapy for compromised skin grafts. In contrast, our review is based on randomized evidence alone, using Cochrane Collaboration methodology. It includes only studies with high internal validity, which offers more robust evidence.

In the present review, two of the included studies were published more than 35 years ago. However, the HBOT technique is merely meant to compress air, and this technology has not changed since then. Hence, we do not think that this time interval affects our conclusions.

One of the included trials reported some major adverse effects in both treatment arms [15]. However, these cannot be explained by a biological rationale and are therefore unlikely to be due to HBOT. From larger descriptive studies, we know that about $20 \%$ of patients experience only mild adverse effects, such as some degree of middle ear barotrauma, and 60 to $70 \%$ of them suffer from measurable, but reversible, worsening myopia [20].

The body of evidence on the usefulness of HBOT is larger for chronic wounds than for acute wounds [6, 8, 21]. Chronic wounds generally have poor local perfusion, which hampers wound healing. This may be counteracted by HBOT. Similarly, with ischemic acute wounds such as crush injuries, HBOT can be effective in overcoming an initial period of local ischemia underlying the problematic healing of these wounds. On the other hand, acute wounds 
are more difficult to treat with HBOT because of their urgency and the limited availability of HBOT facilities. Thus, the application of HBOT can be justified only if the cost is acceptable and HBOT facilities are available at the time of presentation. Hence, it would be bold to advocate the routine use of HBOT for such wounds. In addition, patient selection should be done carefully and according to local guidelines for recognized and reimbursed indications for HBOT [22, 23] (Table 3). On the other hand, HBOT is rather widely available in North America-with more than 300 facilities registered with the Undersea and Hyperbaric Medical Society (UHMS)_Russia, China, and Cuba; it is less well established in Europe and Australasia [24].

Further evaluation through well designed and well conducted RCTs is needed to corroborate clinically relevant effects of HBOT. Patients suitable for HBOT require repeated treatments and depend on availability. These appear to be predominant reasons why RCTs on HBOT, particularly for acute wounds, may be difficult to perform. However, the number of $\mathrm{HBO}$ chambers is increasing, which expands the availability of the treatment. Acute conditions possibly of interest for future studies are flaps used in plastic surgery, calciphylaxis, and jaw surgery because in these circumstances oxygen perfusion can be severely hampered. In such cases, wound healing is at risk, and wound infections are more likely to occur. Currently, an RCT is ongoing to investigate the efficacy of adding HBOT to the treatment of late radiation damage of the lower jaw (osteoradionecrosis) (http: Ilwww.clinicaltrials. gov/, NCT00989820). Present-day reality is that costeffectiveness studies are lacking [25], and transportation logistics may preclude the use of HBOT during an early phase of wound healing of acute surgical and traumatic wounds. Nevertheless, if a HBOT facility is at hand, the

Table 3 Recognized indications for HBOT in the Netherlands (www. cvz.nl)

\begin{tabular}{l}
\hline Acute indications \\
Decompression sickness \\
Air or gas embolism \\
CO poisoning \\
Necrotizing soft tissue infections \\
Crush lesions, compartment syndrome, and other \\
acute traumatic ischemia \\
Skin grafts and flaps (compromised) \\
Limb reimplantation \\
Chronic indications \\
Diabetic ulcer \\
Delayed radiation injury (soft tissue and bony necrosis) \\
Radiocystitis, proctitis, enteritis \\
Osteomyelitis (refractory) \\
\hline
\end{tabular}

evidence found in this review supports its application for acute, difficult to heal wounds.

\section{Conflicts of interest None.}

Open Access This article is distributed under the terms of the Creative Commons Attribution Noncommercial License which permits any noncommercial use, distribution, and reproduction in any medium, provided the original author(s) and source are credited.

\section{References}

1. Lee CK, Hansen SL (2009) Management of acute wounds. Surg Clin North Am 89:659-676

2. Franz MG, Steed DL, Robson MC (2007) Optimizing healing of the acute wound by minimizing complications. Curr Probl Surg 44:691-763

3. Hunt TK, Ellison EC, Sen CK (2004) Oxygen: at the foundation of wound healing-introduction. World J Surg 28:291-293

4. Thackham JA, McElwain DL, Long RJ (2008) The use of hyperbaric oxygen therapy to treat chronic wounds: a review. Wound Repair Regen 16:321-330

5. Wang C, Schwaitzberg S, Berliner E et al (2003) Hyperbaric oxygen for treating wounds: a systematic review of the literature. Arch Surg 138:272-279

6. Kranke P, Bennett M, Roeckl-Wiedmann I et al (2004) Hyperbaric oxygen therapy for chronic wounds. Cochrane Database Syst Rev (2)CD004123

7. Villanueva E, Bennett MH, Wasiak J et al (2004) Hyperbaric oxygen therapy for thermal burns. Cochrane Database Syst Rev (3)CD004727

8. Roeckl-Wiedmann I, Bennett M, Kranke P (2005) Systematic review of hyperbaric oxygen in the management of chronic wounds. Br J Surg 92:24-32

9. Bennett MH, Stanford R, Turner R (2005) Hyperbaric oxygen therapy for promoting fracture healing and treating fracture nonunion. Cochrane Database Syst Rev (1)CD004712

10. Goldman RJ (2009) Hyperbaric oxygen therapy for wound healing and limb salvage: a systematic review. PM R 1:471-489

11. Eskes A, Ubbink DT, Lubbers M et al (2010) Hyperbaric oxygen therapy for acute surgical and traumatic wounds. Cochrane Database Syst Rev (10)CD008059

12. Liberati A, Altman DG, Tetzlaff J et al (2009) The PRISMA statement for reporting systematic reviews and meta-analyses of studies that evaluate healthcare interventions: explanation and elaboration. BMJ 339:b2700

13. Moher D, Liberati A, Tetzlaff J et al (2010) Preferred reporting items for systematic reviews and meta-analyses: the PRISMA statement. Int J Surg 8(5):336-341

14. Higgins JPT, Altman DG (eds) (2008) Cochrane handbook for systematic reviews of interventions, Version 5.0.1 (updated September 2008). The Cochrane Collaboration. http: Ilwww. cochrane-handbook.org

15. Bouachour G, Cronier P, Gouello JP et al (1996) Hyperbaric oxygen therapy in the management of crush injuries: a randomized double-blind placebo-controlled clinical trial. J Trauma 41:333-339

16. Brannen AL, Still J, Haynes M et al (1997) A randomized prospective trial of hyperbaric oxygen in a referral burn center population. Am Surg 63:205-208

17. Hart GB, O'Reilly RR, Broussard ND et al (1974) Treatment of burns with hyperbaric oxygen. Surg Gynecol Obstet 139:693-696 
18. Perrins DJ (1967) Influence of hyperbaric oxygen on the survival of split skin grafts. Lancet 1:868-871

19. Xie ZX, Li CY (2007) Changes in arterial flow after flap grafting under various tensions. Clin Rehabil Tissue Eng Res 11:50045005

20. Leach RM, Rees PJ, Wilmshurst P (1998) Hyperbaric oxygen therapy. BMJ 317:1140-1143

21. Londahl M, Katzman P, Nilsson A et al (2010) Hyperbaric oxygen therapy facilitates healing of chronic foot ulcers in patients with diabetes. Diabetes Care 33:998-1003

22. Ligtenberg G, Kleijnen S, Staal P (eds) (2009) CVZ-HBOT indications (updated February 2009). http: Ilwww.cvz.nl
23. Sheffield PJ, Slade JB (eds) (2010) UHMS Physician CME guidebook. 6th edn (updated January 2010). Undersea and Hyperbaric Medical Society. http:॥www.uhms.org

24. Undersea and Hyperbaric Medical Society (2001) Hyperbaric chambers in North and Central America: a directory of hyperbaric treatment chambers. Undersea and Hyperbaric Medical Society Publications, Durham

25. Ritchie K, Baxter S, Craig J et al (eds) (2008) HTA Programme: Systematic Review 2 (updated July 2008). NHS Quality Improvement Scotland. http:॥www.nhshealthquality.org 